\title{
CLINICO-EPIDEMIOLOGICAL PROFILE OF ACUTE ENCEPHALITIS SYNDROME WITH SPECIAL REFERENCE TO JAPANESE ENCEPHALITIS IN CHILDREN BELOW 12 YEARS OF AGE ADMITTED IN A TERTIARY CARE HOSPITAL OF TRIPURA
}

\author{
Tapash Ghosh1, Nazrul Islam Barbhuiya², Anita Riang Simon³, Sormistha Chowdhury ${ }^{4}$ \\ ${ }^{1}$ Assistant Professor, Department of Paediatrics, Agartala Government Medical College, Agartala. \\ 2Junior Resident, Department of Paediatrics, Agartala Government Medical College, Agartala. \\ $3 J u n i o r$ Resident, Department of Paediatrics, Agartala Government Medical College, Agartala. \\ 4Junior Resident, Department of Paediatrics, Agartala Government Medical College, Agartala.
}

\section{BACKGROUND}

ABSTRACT

Acute Encephalitis Syndrome (AES) is a major public health problem in India. Japanese Encephalitis Virus (JEV) is one of the important causative agents of AES prevailing in this part of the country. It is one of the most important vaccines preventing viral encephalitis in human beings.

The objective of this study is to study aetiology and epidemiology of Acute Encephalitis Syndrome with special reference to Japanese Encephalitis.

\section{MATERIALS AND METHODS}

This was a retrospective descriptive study carried out in the Department of Paediatrics, Agartala Govt. Medical College and GB Pant Hospital, Agartala, Tripura, from 1st December 2015 to 30th November 2016. Hospital records of children admitted with the diagnosis of AES up to 12 years of age were enrolled in the study and were analysed both manually and using SPSS version 15.0 statistical software.

\section{RESULTS}

Out of 104 acute encephalitis cases, $62(59.6 \%)$ were male and $42(40.38 \%)$ were female. The maximum number of AES cases were in the age group of $<5$ years, which was $67.3 \%$. Out of 104 cases of acute encephalitis cases, 20 (19.23\%) were Japanese encephalitis and $2.88 \%$ (3) each of Chikungunya and Dengue. Japanese Encephalitis is most commonly seen in the age group of 6 to 10 years of age (45\%). Majority (65\%) of JE cases presented during the monsoon months of June to August. Most commonly seen in people living in rural area, which was $75 \%$. Case fatality rate for JE was $15 \%$ and sequelae was in $40 \%$ cases as compared with non-JE which was $65 \%$ and $10.71 \%$ respectively.

\section{CONCLUSION}

It has been found that JE remains the most common cause of AES in children in this part of the country with incidence as high as $19.23 \%$ and mortality $15 \%$. It mainly occurs during monsoon season.

\section{KEYWORDS}

Acute Encephalitis Syndrome, Japanese Encephalitis, Neurological Sequelae.

HOW TO CITE THIS ARTICLE: Ghosh T, Barbhuiya NI, Simon AR, et al. Clinico-epidemiological profile of acute encephalitis syndrome with special reference to Japanese encephalitis in children below 12 years of age admitted in a tertiary care hospital of Tripura. J. Evolution Med. Dent. Sci. 2017;6(93):6735-6737, DOI: 10.14260/jemds/2017/1458

\section{BACKGROUND}

Acute Encephalitis Syndrome (AES) remains a major cause for mortality and morbidity. It is characterised by the acute onset of fever with change in mental status. [1] The history of AES in India has paralleled with that of the Japanese encephalitis virus (JEV) since the first report in 1955 from Vellore, Tamilnadu. The first outbreak of JEV was reported in Bankura district, West Bengal in 1973. Thereafter, sporadic cases of AES and outbreaks have been the leading cause of premature deaths due to the disease in India.[2] Viral infections are most common and important cause of

'Financial or Other Competing Interest': None.

Submission 29-09-2017, Peer Review 21-11-2017,

Acceptance 28-11-2017, Published 11-12-2017.

Corresponding Author:

Dr. Nazrul Islam Barbhuiya

Department of Paediatrics,

Agartala Government Medical College,

Agartala, Kunjaban-799006.

E-mail:drnazrul85@yahoo.com

DOI: $10.14260 /$ jemds $/ 2017 / 1458$

\section{(c) (i) $(9)$}

encephalitis. JE and Dengue are more prevalent in South East Asia.[3] Cerebral malaria and non-infectious causes of encephalopathy are required to be excluded while considering AES. Confirmation of diagnosis of JE is usually done by JE specific titers of IgM antibodies in serum and/or in CSF during acute illness of suspected AES case. ${ }^{[4]} \mathrm{JE}$ is the most prevalent and significant mosquito-borne viral encephalitis of man occurring with an estimated 30,000 to 50,000 of cases and 15,000 deaths annually. According to World Health Organisation (WHO) report, approximately 3 billion people are living in countries, which are at risk of JE in Southeast Asia and Western Pacific. About 20\% to 30\% of JE cases are fatal and $30 \%-50 \%$ result in permanent neuropsychiatric sequelae.[5] In the most robust, prospective studies conducted in Western industrialised countries, a minimum incidence of 10.5 per 100,000 AES cases were reported for children and 2.2 per 100,000 for adults. The minimum incidence for all ages was 6.34 per 100,000 from a tropical setting.[6]

In India, the data/ documentation of Directorate of National Vector-Borne Disease Control Programme reflects 
38,979 cases of AES/ 4, 695 cases of JE and 5,908 deaths due to AES/ 810 deaths due to JE in 20 States/ Union territories of India between 2008 - April 2014. Highest number of cases and deaths have been reported from UP, Assam, Bihar, West Bengal and Tamilnadu. [7]

AES prevalence varies from place to place with seasonal variation. In Assam, the cases start appearing from February and attain a peak in the month of July. Due to circulation of enteroviruses, particularly in eastern Uttar Pradesh AES cases are reported round the year.[6,7]

The North Eastern Region of India has been experiencing recurrent episodes of JE with different magnitudes from July to October every year. [8] The present study is carried out with the objective to evaluate the clinico-demographic profile of hospitalised paediatric AES cases to determine the incidence and outcome of acute encephalitis syndrome to document what proportion of these cases are serology proven JE with case fatality.

\section{MATERIALS AND METHODS}

This record based retrospective descriptive study carried out in children up to 12 years with clinical diagnosis of AES admitted in paediatric ward of Agartala Government Medical College and Govind Ballabha Pant Hospital, Tripura, during the study period from $1^{\text {st }}$ December 2015 to $30^{\text {th }}$ November 2016.

During this 1-year period, 104 children admitted who were fulfilling clinical criteria of AES according to WHO case definition (Acute-onset of fever with change in mental status including symptoms such as confusion, disorientation, coma or inability to talk and/or often with new onset of seizures excluding simple febrile convulsion in a person of any age at any time of the year ${ }^{1}$ ) were enrolled in the study, excluding patients presenting with encephalitis but with clinicoinvestigational diagnosis confirmative of cerebral malaria, non-infectious encephalopathy (Hypoxic, ischaemic, vascular, toxic and metabolic) and patients with history of CNS disorder.

Case history sheet, investigation, progress in the hospital and outcome were evaluated and recorded. Serological test for viral marker done by Niv JE MAC-ELISA kit for IgM were used for laboratory test of JE. Data were analysed both manually and using SPSS version 15.0 statistical software, Chi-square test and Fisher's Exact test was used to see significant association. $\mathrm{P}$ value of $<0.05$ was considered as statistically significant.

\section{RESULTS}

During the study period, total number of clinically diagnosed Acute Encephalitis Syndrome patients was 104, which was $3.19 \%$ of total hospitalisation. Out of these 104 cases, 62 (59.6\%) were male and $42(40.38 \%)$ were female. Month wise acute encephalitis cases were shown in Fig. 1. Religion wise- Hindu 90.38\% (94), Muslim 7.44\% (7) and Christian $2.88 \%$ (3) was observed. Majority $71.15 \%$ (74) cases were in ethnic population and $28.84 \%$ (30) in non-ethnic people.

Out of these 104 cases, 20 (19.23\%) were JE and 84 $(80.76 \%)$ were non-JE; among non-JE $2.88 \%$ (3) each of Dengue and Chikungunya was observed. The maximum number of JE cases were reported in the age group of 6 to 10 years, which was $45 \%$ (9), < 5 years' age group $25 \%$ (5) and 11 to 12 years $30 \%$ (6). In Japanese encephalitis, male:female ratio was 1: 1.5. Majority cases of Japanese Encephalitis was observed in June to August (Fig. 1). Majority cases are observed in people living in plain area as compared to hilly area, which was $70 \%(14)$ and $30 \%(6)$ respectively.

Clinically, fever and altered sensorium was the most common presenting feature both in JE and non-JE AES as shown in Fig. 2. In this study, neurological sequelae was more common in JE. It was $40 \%$ as compared with non-JE which was $10.71 \%$, but mortality was more in non-JE as compared with JE, it was $73.8 \%$ and $15 \%$ respectively (Table 1).

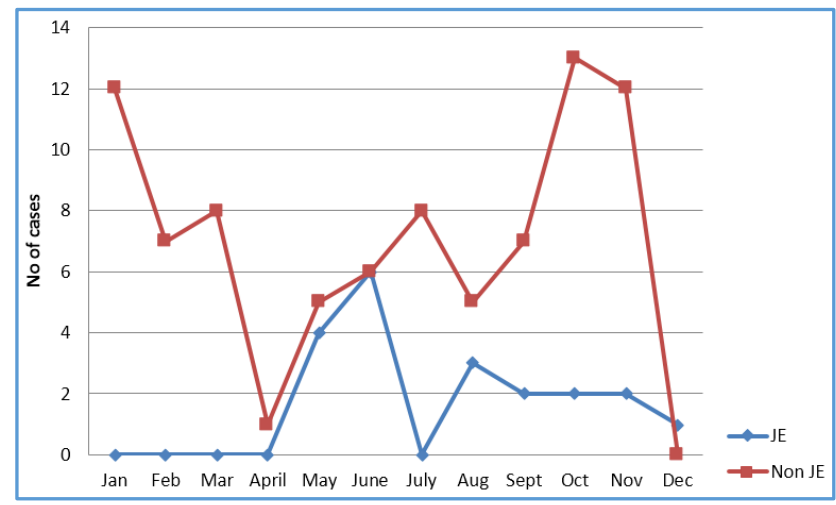

Figure 1. Month Wise Distribution of JE and Non-JE

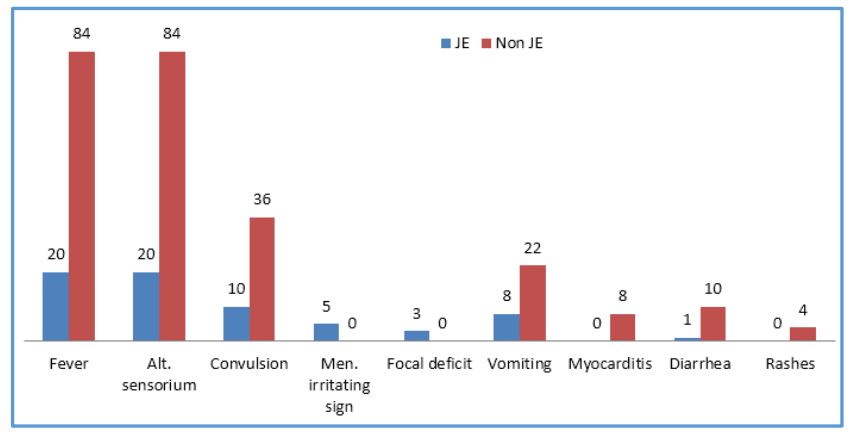

Figure 2. Clinical Features of JE and Non-JE AES

\begin{tabular}{|c|c|c|}
\hline Features & $\begin{array}{c}\text { JE } \\
\text { N (\%) }\end{array}$ & $\begin{array}{c}\text { Non-JE } \\
\text { N (\%) }\end{array}$ \\
\hline Age $(<5$ yrs.) & $5(25)$ & $76(90.47)$ \\
\hline Rural & $15(75)$ & $56(66.66)$ \\
\cline { 2 - 3 } Urban & $5(25)$ & $28(33.33)$ \\
\hline Status epilepticus & $5(25)$ & $15(17.85)$ \\
\hline Hospital stay > 7 days & $13(65)$ & $60(71.42)$ \\
\hline Neurological sequelae & $8(40)$ & $9(11.11)$ \\
\hline Mortality & $3(15)$ & $62(73.80)$ \\
\hline
\end{tabular}

Table 1. Characteristic Features of JE and Non-JE AES

\section{DISCUSSION}

It has been found that incidence of JE is $19.23 \%$ in this study, which is almost similar with other studies done in neighbouring states in Assam and West Bengal.[8,9] The present study did not find any statistically significant sex predilection among AES and JE cases in accordance with other studies by Mohan et al[8] and Bandyopadhyay et al.[9] Although, almost half hospitalisation occurred from people living in hilly area, but maximum JE cases was observed in 
persons living in plains. This might be mosquito habitat and chain of JE virus. Non-JE AES was observed more in patients from hilly areas, might be enteroviruses prevailing in low socio-economical group. Majority of JE cases were seen during monsoon period, peak occurrence in July. Phukan et al,[10] Mohan et al,[8] Borkotoki et al[11] and Patgiri et al[12] reported similar findings might be due to increase in mosquito population leading to JE during the monsoon season. Age wise maximum JE reported more than 5 years, but non-JE observed more cases in less than 5 years ( $p$ value $=.000$ ).

Our study shows fever and altered sensorium as the commonest presenting features present in all JE cases. Similar observations were made by Parida et al[13] and Sengupta et al.[14] In this study, it was observed that mortality was $15 \%$ and $73.8 \%$ in JE and non-JE AES. High mortality in non-JE AES might be lower age of presentation.

As this is a retrospective descriptive study, hospital course, symptomatology etc. could not be evaluated properly. Moreover, size of the study is also small to represent the whole scenario. These need further study.

\section{CONCLUSION}

It has been found that JE remains the most common cause of AES in children in this part of the country with incidence as high as $19.23 \%$ and mortality $15 \%$. It mainly occurs during monsoon season. Concrete efforts need to be made to reduce disease burden by improving and strengthening vector control methods and public awareness programme. Vaccines offer the most promising tool for preventing morbidity and mortality caused by Japanese encephalitis.

\section{REFERENCES}

[1] Solomon T, Thao TT, Lewthwaite P, et al. A cohort study to assess the new WHO Japanese encephalitis surveillance standards. Bull World Health Organ 2008;86(3):178-86.

[2] Joshi R, Kalantri SP, Reingold A, et al. Changing landscape of acute encephalitis syndrome in India: a systematic review. Natl Med J India 2012;25(4):21220.

[3] Misra UK, Tan CT, Jayantee K. Seizures in encephalitis. Neurology Asia 2008;13:1-13.
[4] Center for Disease Control and Prevention (CDC). Question and answer about Japanese Encephalitis March 2013.

http://www.cdc.gov/japaneseencephalitis/qa/index.h tml. Assessed on 12.2.2010.

[5] Dutta P, Khan SA, Khan AM, et al. The effect of insecticide-treated mosquito nets (ITMNs) on Japanese encephalitis virus seroconversion in pigs and humans. Am J Trop Med Hyg 2011;84(3):466-72.

[6] National Vector Borne Disease Control Programme. Guidelines for Surveillance of Acute Encephalitis [Internet]. Delhi: Publication of Govt. of India; August 2009. Available from:

nvbdcp.gov.in/Doc/AES\%20guidelines.pdf.

[7] National Vector Borne Disease Control Programme. Revised Treatment Guidelines for AES including JE [Internet]. Delhi: Publication of Govt. of India. August 2009. Available from:

www.nvbdcp.gov.in/Doc/Revised\%20guidelines\%20o n\%20AES_JE.pdf.

[8] Mohan DG, Hazarika NK. A clinico-pathological study and demographic profile of Japanese encephalitis from a tertiary care hospital in Assam, India. Int J Curr Microbiol App Sci 2015;4(6):522-9.

[9] Bandyopadhyay B, Bhattacharyya I, Adhikary S, et al. Incidence of Japanese encephalitis syndrome among acute encephalitis syndrome cases in West Bengal, India. Biomed Research International Article ID: 896749, 2013;2013:5.

[10] Phukan AC, Borah PK, Mahanta J. Japanese encephalitis in Assam, northeast India. Southeast Asian J Trop Med Public Health 2004;35(3):618-22.

[11] Borkotoki U, Borkotoki S, Barua P, et al. Japanese encephalitis (JE) among acute encephalitis syndrome (AES) cases-a hospital based study from upper Assam, India. Int J of Health Sci and Res 2016;6(5):72-7.

[12] Patgiri SJ, Borthakur AK, Borkakoty B, et al. An appraisal of clinicopathological parameters in Japanese encephalitis and changing epidemiological trends in upper Assam, India. Indian J Pathol Microbiol 2014;57(3):400-6.

[13] Parida M, Dash PK, Tripathi NK, et al. Japanese encephalitis outbreak, India, 2005. Emerg Infect Dis 2006;12(9):1427-30.

[14] Gupta SN, Sen MK, Das PK, et al. Clinical profile of the epidemic of Japanese encephalitis at Bankura. Indian J Med Res 1976;64(10):1393-402. 